\title{
Sustentabilidade e Intercompreensão: Perspetivas e contributos de um centro de investigação em educação
}

\author{
Patrícia Sá, J. Bernardino Lopes \\ \& Isabel P. Martins
}

Resumo:

\begin{abstract}
A adoção, pelas Nações Unidas, da Agenda 2030 para o Desenvolvimento Sustentável levanta novos desafios ao campo da investigação em educação. No contexto dos atuais desafios sócio-económico-ambientais e das questões internacionais relacionadas como desenvolvimento sustentável é um imperativo moral que os investigadores contribuam para a concretização desta resolução. Neste artigo apresentamos dados provenientes de um estudo realizado sobre as perceções que investigadores seniores com responsabilidade na definição da política científica de um centro de investigação em educação têm sobre os conceitos de Sustentabilidade e de Intercompreensão. A metodologia seguida insere-se num paradigma interpretativo. Os resultados indicam que alguns dos investigadores participantes revelam um pensamento estruturado sobre estes conceitos, reconhecendo a sua centralidade na definição da investigação em educação para o séc. XXI. No entanto, configura-se a necessidade de um aprofundamento de ideias e consequente reorientação na conceção e consecução da agenda de investigação no futuro próximo.
\end{abstract}

Palavras-chave:

sustentabilidade; intercompreensão; investigação em educação; agenda 2030 para o desenvolvimento sustentável 


\title{
Sustainability and Intercomprehension: Perspectives and Contributions of an Education Research Center
}

\begin{abstract}
The adoption of the 2030 Agenda for the Sustainable Development by the United Nations (UN) raises new challenges for the educational research field. In the context of current socio-economic/environmental challenges and international sustainable development issues it is a moral imperative for researchers to contribute to the fulfillment of this resolution. In this paper we report data from a study within which we investigated the perceptions about the concepts of Sustainability and Intercomprehension of senior educational researchers with responsibility in defining the scientific policy of a research centre in education. The methodology followed is inserted in an interpretative paradigm. The results indicated that some of the participating researchers have structured and reasoned thinking on these concepts, recognizing their centrality in the definition of the educational research for the XXI century. However, the need for a deepening of ideas and consequent reorientation in the conception and attainment of the research agenda in the near future is configured.
\end{abstract}

Keywords: sustainability; intercomprehension; research in education; 2030 agenda for sustainable development.

\section{Sostenibilidad e Intercomprensión: Perspectivas y contribuciones de un centro de investigación en educación}

Resumen: La adopción por las Naciones Unidas de la Agenda 2030 para el Desarrollo Sostenible plantea nuevos desafíos al campo de la investigación en educación. En el contexto de los actuales desafíos socioeconómicoambientales y de las cuestiones internacionales relacionadas como desarrollo sostenible es un imperativo moral que los investigadores contribuyan a la concreción de esta resolución. En este artículo presentamos datos provenientes de un estudio realizado sobre las percepciones que investigadores mayores con responsabilidad en la definición de la política científica de un centro de investigación en educación tienen sobre los conceptos de Sostenibilidad e Intercomprensión. La metodología seguida se inserta en un paradigma interpretativo. Los resultados indican que algunos de los investigadores participantes revelan un pensamiento estructurado sobre estos conceptos, reconociendo su centralidad en la definición de la investigación en educación para el s. XXI. Sin embargo, se configura la necesidad de una profundización de ideas y consecuente reorientación en la concepción y consecución de la agenda de investigación en el futuro próximo.

Palabras clave: sostenibilidad; intercomprensión; investigación en educación; agenda 2030 para el desarrollo sostenible.

\section{Durabilité et Intercompréhension: perspectives et contributions d'un centre de recherche en éducation}

Résumé : L'adoption par les Nations Unies du Programme de développement durable à l'horizon 2030 pose de nouveaux défis dans le domaine de la recherche en éducation. Dans le contexte des défis socio-économiques et environnementaux actuels et des questions internationales connexes telles que le développement durable, il est impératif que les chercheurs contribuent à la mise en œuvre de cette résolution. Dans cet article, nous présentons les données d'une étude réalisée sur les perceptions que les chercheurs seniors responsables de la définition de la politique scientifique d'un centre de recherche en éducation ont sur les concepts de durabilité et d'intercompréhension. La méthodologie est insérée dans un paradigme interprétatif. Les résultats indiquent que certains des chercheurs participants présentent une réflexion structurée sur ces concepts, reconnaissant leur centralité dans la définition de la recherche en éducation pour le XXI siècle. Cependant, la nécessité d'un approfondissement des idées et d'une réorientation conséquente dans la conception et la réalisation du programme de recherche dans un avenir proche est configurée.

Mots-clés: durabilité; intercompréhension; recherche en éducation; agenda 2030 pour le développement durable. 


\section{Introdução}

Sustentabilidade e Intercompreensão (IC) são conceitos que do ponto de vista académico, das agendas de financiamento da investigação e da ligação da investigação à sociedade, se impõem por implicarem uma reconceptualização da investigação em educação e a emergência de um novo paradigma, marcado por uma preocupação mais ampla, mais humanista e menos funcional do seu futuro. Os referidos conceitos exprimem, aliás, domínios que se tornaram temas da agenda de desenvolvimento à escala global e reconhecidos como orientações estratégicas para a intervenção dos mais diversos atores em todos os domínios de ação.

O percurso feito desde a Década das Nações Unidas da Educação para o Desenvolvimento Sustentável (DNUEDS, 2005-2014) até à resolução "Transforming our world: the 2030 Agenda for Sustainable Development" (A/RES/70/1) e aos Objetivos para o Desenvolvimento Sustentável, evidencia o reconhecimento, por parte das Nações Unidas e da UNESCO, da urgência de um compromisso global para com a Sustentabilidade e IC, e da importância que a educação assume enquanto área prioritária de ação.

Adicionalmente, a identificação dos investigadores e educadores como agentes principais de intervenção e transformação; e a constatação da necessidade de mais investigação no domínio da Educação para o Desenvolvimento Sustentável (ESD), e de mais investimento na formação de professores, são inegáveis evidências desta valorização.

Contudo, trabalhar em prol da Agenda 2030 exige à comunidade uma reflexão profunda no sentido de novas formas de conceptualizar a educação e a investigação e de fazer emergir um novo paradigma para a investigação em educação orientado para os objetivos da Sustentabilidade e da IC. A intervenção dos investigadores dependerá do entendimento que têm sobre estes objetivos e sobre a sua pertinência na investigação em educação e nos domínios a que se estende o seu impacte. Neste sentido, consideramos fundamental perceber qual o entendimento que investigadores em educação, seniores e com responsabilidade na política educativa de um centro de investigação, têm sobre os conceitos de Sustentabilidade e IC, fazendo disso a questão central no estudo que fundamenta o artigo que se apresenta.

Neste artigo apresenta-se uma clarificação conceptual e terminológica dos conceitos de Sustentabilidade e IC. De seguida, faz-se uma apresentação da metodologia utilizada e apresenta-se uma síntese e conclusão do estudo.

\section{Sustentabilidade e Intercompreensão: clarificação terminológica e conceptual}

Sustentabilidade e IC são conceitos polissémicos, complexos e dinâmicos, características que acentuam a imprecisão terminológica e conceptual que se pode encontrar na literatura de referência. Não sendo objetivo deste artigo apresentar uma revisão exaustiva 
destes conceitos, consideramos importante evidenciar, por um lado, esta proliferação de entendimentos enquanto condição indutora da referida imprecisão conceptual e, por outro, as principais ideias-chave presentes em alguma da literatura de referência.

Nas últimas décadas, as referências aos conceitos de Desenvolvimento Sustentável (DS) e Sustentabilidade surgem na literatura dos mais diversos domínios, sendo a sua utilização transversal aos vários sectores e áreas da sociedade (Amador \& Oliveira, 2013; Faustino \& Amador, 2016; Sartori, Silva \& Campos, 2014). Vários foram os autores que, ao longo dos anos, apresentaram tentativas de definição e sistematizações sobre estes conceitos (Amador \& Oliveira, 2013; Bettencourt \& Kaur, 2011; Faustino \& Amador, 2016; Giovannoni \& Fabietti, 2014; Sartori, Silva \& Campos, 2014). A sua natureza camaleónica e multidimensional é amplamente evidenciada na literatura (Drexhage \& Murphy, 2010; Giovannonni \& Fabietti, 2014) e é explícita nos vários documentos orientadores que a UNESCO tem vindo a publicar nos últimos anos (UNESCO, 2005, 2012, 2014, 2017). As dimensões mais unanimemente reconhecidas são as ambiental, social e económica, embora outras possam ser identificadas.

A distinção entre os conceitos de DS e de Sustentabilidade e a natureza da sua relação também tem vindo a ser explorada. A sustentabilidade pode ser definida como a capacidade de um sistema humano resistir ou se adaptar indefinidamente às mudanças que vão ocorrendo (Faustino \& Amador, 2016). Para os mesmos autores, o DS é uma forma intencional de mudança que mantém, ou aumenta, este atributo do sistema, tendo em consideração as necessidades da população. Assim, o DS é percebido como forma ou meio para alcançar a Sustentabilidade, esta tida como meta (Faustino \& Amador, 2016).

Face à proliferação de entendimentos consideramos pertinente o conceito apresentado por Constanza e Patten (1995, p.31), para quem

... sustainability is a relationship between dynamic human economic systems and dynamic, but slower, ecological systems, in which: a) human life can develop indefinitely; b) human individuals can flourish; c) human culture can develop and d) effects of human activities remain within bounds so as not to destroy the diversity, complexity and functioning of the ecological life-support system.

O conceito de IC é, também, um conceito que tem vindo a evoluir, sendo hoje considerado, no campo da Didática das Línguas (DL), um meta-conceito (Melo \& Santos, 2007).

De acordo com Araújo e Sá (2012), este conceito terá de ser entendido à luz das sociedades plurais em que habitamos. A IC alinha-se com as grandes finalidades da educação, como as de paz, coesão social, cidadania e diálogo intercultural (idem), configurando-se como um conceito entendido numa perspetiva humanista, mais comprometido e mais atuante (Alarcão \& Araújo e Sá, 2010). 
Dada a sua complexidade e polissemia, este conceito tem sido alvo de múltiplas abordagens e sistematizações (Ferrão, Tavares, Silva \& Silva e Silva, 2010; Pinho \& Andrade, 2011). Araújo e Sá (2012) propõe uma sistematização dos eixos temáticos transversais encontrados por diferentes autores em alguns dos trabalhos mais significativos realizados neste domínio. Assim, a autora destaca alguns entendimentos da IC: i) atributo, capacidade, aptidão e/ou competência do sujeito, que se manifesta na prática social; ii) finalidade e meio de educação; iii) abordagem didática, modo de ensinar e aprender línguas ou metodologia de ensino; iv) prática, fenómeno e; v) estratégia de comunicação.

Para referência consideramos o conceito proposto pelo Ministério da Cultura e da Comunicação francês (2015, p.3):

L'intercompréhension est une forme de communication plurilingue où chacun comprend les langues des autres et s'exprime dans la ou les langue(s) qu'il maîtrise, instaurant ainsi une équité dans le dialogue, tout en développant, à différents niveaux, la connaissance de langues dans lesquelles on a des compétences de réception (c'est à dire de compréhension) et non de production.

\section{Metodologia}

No estudo que se apresenta pretendeu-se analisar as perceções sobre os conceitos de Sustentabilidade e de IC de investigadores em educação, seniores e com responsabilidades na definição da política científica de um Centro de Investigação em Educação (CIE). Este CIE encontra-se numa fase de reestruturação e reorganização das suas estruturas de investigação. Neste processo, Sustentabilidade e IC foram assumidos pela coordenação como conceitos-chave, transversais à investigação que aí se desenvolve, pelo que a identificação e análise das perceções que os membros do CIE têm sobre estes conceitos surge como fundamental para o Centro e justifica a opção dos investigadores para a realização do estudo.

Metodologicamente, optou-se por uma estratégia "descritivo-interpretativa", de natureza exploratória, que, como diz Amado (2017, p. 120), se realiza "sobre uma 'amostra' não estatística de sujeitos (que têm em comum determinada particularidade ou experiência de vida, sendo por isso socialmente representativos)". Como afirma o autor,

Não se tratará propriamente de estudos de caso, por Ihes faltar uma verdadeira e necessária contextualização; nem se tratará de estudo biográfico, por se limitar a aspetos muito circunscritos da experiência de vida dos sujeitos entrevistados; contudo, nestes estudos descritivointerpretativos está sempre 
presente a preocupação por descrever padrões e características de uma dada população ou área de interesse (Amado, 2017, p. 120).

A recolha de dados foi feita através de entrevista individual semiestruturada (Bogdan \& Biklen, 2013) a treze sujeitos que foram escolhidos para participar neste estudo por reunirem um conjunto de características comuns relevantes para o estudo: todos são investigadores seniores, doutorados há mais de dez anos, coordenadores de, pelo menos, uma das estruturas em que o CIE se organiza e com responsabilidades na definição da política científica do Centro.

Estes sujeitos serão referidos pelo código definido para cada um deles (P1, P2, ..., P13). As tabelas que se seguem sistematizam as principais características destes sujeitos no que diz respeito à conclusão do doutoramento e à área de investigação.

\begin{tabular}{|c|c|}
\hline Conclusão Doutoramento (anos completos) & Sujeitos \\
\hline $10-15$ & P1; P10 \\
\hline $16-20$ & P2; P5; P6; P9; P11 \\
\hline $21-25$ & P4; P7; P8; P13 \\
\hline $26-30$ & P3; P12 \\
\hline
\end{tabular}

Figura 1. Distribuição dos sujeitos por anos de conclusão do doutoramento

\begin{tabular}{|c|c|}
\hline Área de investigação & Sujeitos \\
\hline Educação em Ciências & P1; P3; P4; P6; P10; P11; P12 \\
\hline Educação em Línguas & P2; P5; P8; P13 \\
\hline Ciências da Educação & P9; P7 \\
\hline
\end{tabular}

Figura 2. Distribuição dos sujeitos por área de investigação

O guião da entrevista foi definido considerando a questão de investigação e respetivos objetivos. A validação foi realizada por um painel de seis investigadores seniores que se pronunciaram sobre a estrutura e tipologia do guião apresentado, e sobre a formulação das questões e a sua adequação aos objetivos do estudo definidos.

O guião da entrevista está organizado em três partes. As Partes I e III são de apresentação do projeto de investigação e de finalização da entrevista, respetivamente. A Parte ॥ teve como principais objetivos identificar as perceções dos sujeitos entrevistados sobre: i) os conceitos de IC e de Sustentabilidade; ii) os projetos de investigação que os sujeitos relacionam com Sustentabilidade e IC; iii) a articulação entre as estruturas do CIE em análise.

Cada um dos participantes foi entrevistado individualmente, sem a presença de outras pessoas, no local que definiu, de acordo com a sua disponibilidade e sem ter 
conhecimento das posições assumidas pelos outros entrevistados. As respostas expressas traduzem as ideias próprias de cada investigador. As entrevistas tiveram uma duração média de 60 minutos, foram audiogravadas e posteriormente transcritas.-

A análise de conteúdo foi realizada com base num conjunto de procedimentos sistemáticos, interpretativos e inferenciais aplicados ao conteúdo de cada entrevista (Bardin, 2014), e que possibilitaram a construção das categorias de análise. Este procedimento implicou: i) uma etapa de pré-análise, que consistiu numa leitura flutuante das transcrições das entrevistas com o objetivo de perceber a estrutura e organização de cada entrevista e identificar tendências e padrões relevantes entre as várias entrevistas (Bardin, 2014); ii) operações de "recorte de texto" em unidades comparáveis para posterior codificação; iii) e uma etapa de exploração de todo o material reunido com o intuito da sua codificação e categorização. Nesta última etapa procedeu-se à divisão do texto em unidades de sentido e à atribuição de um código a cada uma destas unidades (Bardin, 2014; Costa \& Amado, 2018). O procedimento de recorte, codificação e reorganização do corpus foi permitindo a identificação das categorias de conteúdo para cada dimensão de análise. A análise foi realizada com recurso ao software webQDA (www.webqda.net).

Na sua versão final, a matriz de análise de conteúdo das entrevistas integra duas dimensões: Dimensão Sustentabilidade (D_S) e Dimensão Intercompreensão (D_Int). Cada uma destas Dimensões está organizada em três categorias de análise não exclusivas (Fig. 1).

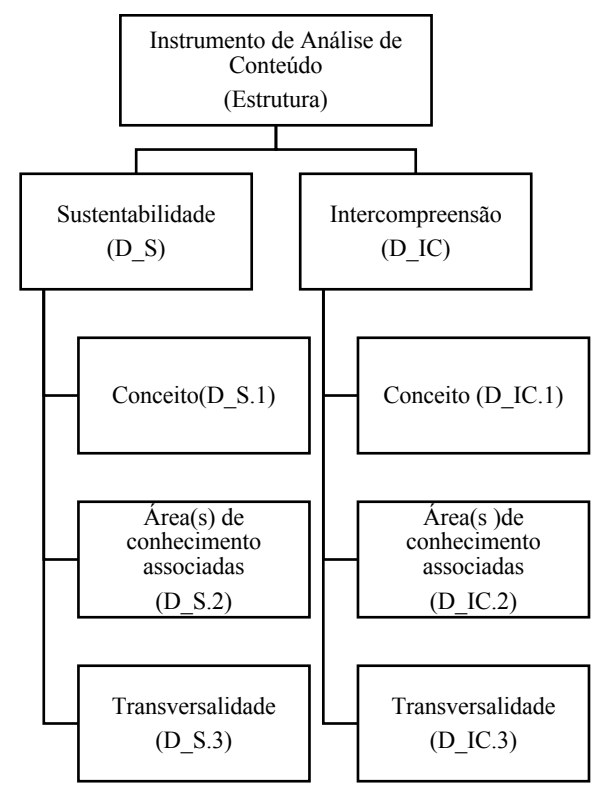

Figura 3. Dimensões e categorias de análise do Instrumento de Análise de Conteúdo das entrevistas 


\section{Apresentação dos resultados}

Nesta secção apresenta-se, por dimensão de análise, uma sistematização das principais ideias que emergiram da análise de conteúdo conduzida a cada uma das entrevistas realizadas. Esta apresentação será feita em forma de tabela, uma para cada categoria de análise.

\section{Dimensão Sustentabilidade (D_S)}

Resultados para a Categoria Sustentabilidade - Conceito (D_S.1)

\begin{tabular}{|c|c|}
\hline \multicolumn{2}{|c|}{ D_S.1 } \\
\hline $\begin{array}{c}\text { Conceito de Sustentabilidade entendido } \\
\text { como } \ldots\end{array}$ & Investigadores entrevistados \\
\hline $\begin{array}{c}\text { Multidimensional } \\
\text { Dimensão ambiental }\end{array}$ & P1, P3, P4, P5, P6, P8, P9, P10 e P11 \\
\hline Dimensão social & P3, P4, P5, P6, P8, P10, P11 \\
\hline Dimensão cultural & P3, P4, P5, P6, P8, P10, P11 \\
\hline Dimensão académica & P8 e P9 \\
\hline Polissémico & P1, P2, P3, P9 e P12 \\
\hline Complexo & P11, P12 e P13 \\
\hline Perspetiva de futuro & P1, P2, P3, P4, P5, P6, P7, P8, P9, P10, P10, \\
\hline Valor & P11, P12 e P13 \\
\hline Abordagem educativa & P4, P6 e P7 P10 e P13 \\
\hline Princípio orientador da ação educativa e da \\
investigação em educação
\end{tabular}

Figura 4. Ideias-chave sobre o conceito de sustentabilidade

A ideia de multidimensionalidade do conceito é comum a nove dos treze sujeitos. Embora a maioria entenda o conceito de sustentabilidade como multidimensional, as dimensões identificadas não são comuns a todos. As dimensões do conceito mais referidas são as dimensões ambiental e social (entendimento presente em sete dos nove casos).

Para P4, por exemplo, a sustentabilidade «... é algo que pretendemos atingir num mundo em que nos situamos, em que tudo pode ser esgotável...». No mesmo sentido, P5 refere a ideia de que «... temos de nos preocupar com a sustentabilidade dos contextos 
... e do ambiente e do planeta...». Já para P6 o entendimento do conceito não se restringe a «... alterações climáticas e coisas do género,..... Para o sujeito, a sua perceção deste conceito é feita «... muito numa dimensão social», de preocupação com o outro.

$\mathrm{Na}$ análise realizada emergiu, ainda, a consideração de uma dimensão académica do conceito, relacionada com os processos de investigação, que foi definida por P9 como «... a possibilidade de continuar investigação em curso». Esta ideia de continuidade está presente, também, em P12, para quem a sustentabilidade da investigação «... tem mais a ver com o facto de a investigação [...] ter algum impacto [...] quando os projetos estão a ser desenvolvidos ... ». A sustentabilidade da investigação é, ainda, associada ao impacte, mesmo que temporalmente limitado.

Em todos os casos analisados se replica a ideia de que o conceito de sustentabilidade é um conceito polissémico e complexo. Esta ideia é explicitamente evidenciada por P3: «Eu penso que relativamente ao conceito de sustentabilidade existem... designações correntes, quer dizer, é um termo que na linguagem corrente tem significados que não são o significado científico que agora lhe queremos atribuir, ou o significado que prevalece em trabalhos de investigação, ou que é linguagem de investigadores...». O sujeito distingue entre o entendimento académico do conceito e aquele que é mais próprio de uma linguagem corrente, do senso comum. No domínio da investigação em educação em particular, P3 considera que «... o termo tem significado próprio, e esse significado depende, também dos quadros referenciais que os autores tomam.» Para o sujeito, «... podemos utilizar a mesma terminologia, mas não Ihe atribuímos o mesmo significado, nem lhe damos a mesma importância».

A perceção de sustentabilidade numa perspetiva de futuro é explicitamente referida em cinco dos sujeitos entrevistados. Para P5, por exemplo, a sustentabilidade «... é a grande finalidade da educação [...] se pensarmos que a educação serve para construir sociedades melhores e um mundo melhor».

Embora a análise de conteúdo das entrevistas tenha permitido inferir que todos os sujeitos consideram a sustentabilidade um valor e que o relacionam com questões de solidariedade e justiça, apenas três são explícitos nesta formulação. Esta ideia é muito clara em P6, que perceciona a sustentabilidade como sinónimo de «... atender ao outro, muito de respeitar o outro, muito de sabermos conviver da melhor maneira possível para tornar este planeta, de facto, sustentável».

Em três dos entrevistados é possível perceber o conceito de sustentabilidade enquanto abordagem educativa. Para P11, a sustentabilidade «...é um conceito ligado ao desenvolvimento sustentável e, nessa medida, à educação para o desenvolvimento sustentável...». Para o mesmo sujeito, «... num mundo complexo [...] com desenvolvimentos humanos muitos díspares, teríamos um desenvolvimento insustentável [...] que carece de uma educação que problematiza estas questões, que tenha sobre elas também uma visão no contexto de educação formal...». 
A ideia de sustentabilidade enquanto princípio orientador da investigação e da ação é encontrada de forma explícita em dois entrevistados. Para P7 «... as questões da sustentabilidade não podem deixar de estar presentes [...] porque são princípios, valores que norteiam a nossa investigação, a nossa ação nos contextos, no sentido de criar uma sociedade $[\ldots]$ que respeita o outro...».

Resultados para Categoria Sustentabilidade - Áreas de conhecimento associadas (D_S.2)

\begin{tabular}{|c|c|}
\hline \multicolumn{2}{|c|}{ D_S.2 } \\
\hline Áreas de conhecimento associadas & Investigadores entrevistados \\
\hline Ciências & P7, P9 e P12 \\
\hline Línguas & P8 \\
\hline
\end{tabular}

Figura 5. Áreas de conhecimento associadas ao conceito de sustentabilidade

Para a categoria D_S.2, é possível verificar que a maioria dos sujeitos não faz uma associação do conceito de sustentabilidade a uma área de conhecimento em particular. No entanto, P7, P9 e P12 relacionam o conceito à área das ciências. P9, por exemplo, considera que o conceito de sustentabilidade espelha a dominância de um dos grandes grupos de investigação do CIE, relacionando-o com o seu domínio de investigação: «... as duas linhas espelham... digamos ... a dominância de dois grandes grupos dentro do centro, não é? Um grupo mais orientado para as questões da sustentabilidade no sentido [...] mais das ciências, das biologias, das ciências naturais, das físicas,...».

Resultados para Categoria Sustentabilidade - Transversalidade (D_S.3)

\begin{tabular}{|c|c|}
\hline \multicolumn{2}{|c|}{ D_S.3 } \\
\hline Transversalidade & Investigadores entrevistados \\
\hline Conceito transversal ao CIE & P3, P4, P6, P7 e P13 \\
\hline Conceito não transversal ao CIE & P1, P2 e P9 \\
\hline $\begin{array}{c}\text { Conceito transversal enquanto meta } \\
\text { educativa }\end{array}$ & P2, P5, P9, P11, P12 e P13 \\
\hline
\end{tabular}

Figura 6. Ideias sobre a transversalidade do conceito de sustentabilidade

Para a categoria D_S.3, é possível distinguir duas abordagens à transversalidade do conceito:

- o entendimento da transversalidade do conceito de sustentabilidade dentro do CIE; embora tal posição possa não ser clara para todos os membros desta unidade de investigação, por constrangimentos diversos. Esta questão é explicitada por P13, que 
percebe a transversalidade do conceito pelas interseções que este permite: «... eu acho que elas existem, acho que já vamos começando a nos aperceber coletivamente dessas intersecções, mas acho que ainda há muito trabalho que nós temos que fazer e que ainda não fizemos. [...] Acho que já há muitas interações que estão ligadas, agora, não posso dizer que o centro assuma essas interações de uma forma coletiva, que seja uma coisa que toda a gente sente.».

- a transversalidade do conceito é referido como meta educativa, ou conceito-chave face aos problemas societais atuais. Para P9, considerar a sustentabilidade a partir de uma dimensão mais social, mais política, permite a sua consideração enquanto «... exercício, preocupação de todos, quer dizer, isso retira especificidade à investigação e [...] nesse sentido todos podem reclamar, digamos assim, a sua quota-parte de intervenção nesta sustentabilidade...».

- para três dos inquiridos este não é um conceito transversal ao ClE. A propósito da explicitação sobre a sua perceção do conceito de sustentabilidade, P1 refere «[...] percebo sustentabilidade no seu sentido amplo [...] quer da investigação, quer a sustentabilidade do conhecimento que vamos produzindo, quer no âmbito mais específico da educação para o desenvolvimento sustentável [...] E, portanto, percebo, entendo e sinto que ainda temos que dar grandes passos para que ele seja verdadeiramente transversal, porque não é.».

\section{Dimensão Intercompreensão (D_IC)}

Tal como para a Dimensão Sustentabilidade, a análise realizada permitiu identificar as ideias que se replicam para a Dimensão IC.

Resultados para Categoria IC - Conceito (D_IC.1)

\begin{tabular}{|c|c|}
\hline \multicolumn{2}{|c|}{ D_IC.1 } \\
\hline IC entendida como $\ldots$ & Investigadores entrevistados \\
\hline Capacidade interna do sujeito & P2, P13 e P10 \\
\hline Abordagem didática & P10 e P13 \\
\hline $\begin{array}{c}\text { Possibilidade de entendimento e/ou processo } \\
\text { de comunicação }\end{array}$ & P1, P2, P4, P5, P6, P10 e P11 \\
\hline Valor & P3, P5, P7, P12 e P13; \\
\hline Conceito multidimensional & P1, P2, P3, P4, P5, P6, P7, P8, P9, P10, \\
\hline P11, P12 e P13
\end{tabular}

Figura 7. Ideias-chave para o conceito de IC 
Para a categoria D_IC.1, em três das entrevistas analisadas é possível encontrar um entendimento do conceito de IC enquanto capacidade interna do sujeito, competência desenvolvida ou a desenvolver. Na sua explicitação sobre o conceito, P13 refere que a IC pode ser entendida como «... uma competência, ou seja, como a capacidade que nós todos temos de construir sentido em diferentes línguas, sem termos de passar por línguas francas e por línguas comuns.» No mesmo sentido, P10 refere que «... a ideia principal quando se fala na aprendizagem de línguas com a mesma raiz, se pode aprender uma língua estrangeira [...] mobilizando os conhecimentos e as competências que tem na língua materna».

Para P10 e P13 a IC pode ser vista como uma abordagem didática, uma forma de intervenção em contextos formais de ensino e de aprendizagem com estratégias e recursos próprios. "Depois, em termos didáticos, é a ideia de que este conceito pode ser didatizado, ou seja, podemos inclui-lo no discurso didático a partir de uma determinada forma de organizar os processos de ensino e aprendizagem desde logo das línguas.» (P13)

A ideia do conceito enquanto possibilidade de entendimento (entre pessoas, culturas, religiões, línguas, domínios de trabalho...) e/ou processo de comunicação é uma das mais replicadas, como se pode perceber no discurso de P13: «... o conceito de intercompreensão é um conceito rentável porque foi definido de múltiplas formas [...] e um conceito útil porque colocou o processo de compreensão de falantes de diferentes línguas, ou da mesma língua, como um processo que é possível construir independente do repertório dos sujeitos [...]».

A IC como valor é explicitamente referida por cinco dos trezes sujeitos. Para P12 o conceito de IC é um conceito «... fundamental para a sociedade atual... para os desafios que a sociedade vive hoje e que se julga que viverá amanhã [...] e, portanto, vejo-o como valor».

Embora seja possível inferir que todos os sujeitos consideram a IC um conceito complexo e multidimensional, apenas P8, P9 e P13 fazem referência explícita a este denominador do conceito. P8 refere-o como um conceito "... que foi evoluindo e alargando a sua abrangência". Para P13, este é um conceito que «... tem vindo a ganhar [...] uma grande polifonia em termos concetuais, teóricos, [...] e há mesmo redes internacionais onde o conceito é discutido em várias vertentes.». 
Resultados para Categoria Intercompreensão - Áreas de conhecimento (D_IC.2)

\begin{tabular}{|c|c|}
\hline \multicolumn{2}{|c|}{ D_IC.2 } \\
\hline Áreas de conhecimento associadas & Investigadores entrevistados \\
\hline Ciências & P3 \\
\hline Línguas & P3, P6, P7, P8, P9, P12 e P13 \\
\hline
\end{tabular}

Figura 8. Ideias sobre a transversalidade do conceito de IC

A maioria dos sujeitos relaciona a IC com o domínio das Línguas. P7, por exemplo, relaciona o conceito às «... palavras-chave do Gl que se ocupa das questões da diversidade linguística e cultural». Para P6 esta perceção do conceito acompanha um entendimento de uma evolução na $I C$ «... porque eu sei que intercompreensão surgiu num contexto muito restrito das línguas e muito focado no compreender o outro...».

Resultados para Categoria Intercompreensão - Transversalidade (D_IC.3)

\begin{tabular}{|c|c|}
\hline \multicolumn{2}{|c|}{ D_IC.3 } \\
\hline Transversalidade & Investigadores entrevistados \\
\hline Conceito transversal ao CIE & P1, P2, P3, P4, P6 P7, P12 e P13 \\
\hline $\begin{array}{c}\text { Conceito transversal enquanto meta } \\
\text { educativa }\end{array}$ & P2, P5, P6, P10 e P11 \\
\hline
\end{tabular}

Figura 9. Ideias sobre a transversalidade do conceito de IC

Relativamente à categoria D_IC.3, o entendimento da transversalidade do conceito é feito:

- ou por referência ao CIE. P1, P2, P3, P4, P7, P12 e P13 percebem-no como conceito transversal à unidade de investigação a que pertencem. Para P4 é um «... conceito transversal, um diretor de ideias [...] e nós temos de ir olhando para aquilo que fazemos e tentando ver de que forma específica é que isto se vai aproximando deste conceito geral...»;

- ou por referência à IC enquanto finalidade educativa e que, por essa razão, é transversal à investigação em educação: «O nosso centro é primeiramente um centro de investigação em educação, e portanto não me parece que faça sentido nós tentarmos produzir conhecimento sobre [...] educação sem que este termo direta ou indiretamente, explícita ou implicitamente lá esteja...» (P6). 


\section{Síntese e conclusões}

O estudo realizado permitiu perceber, relativamente ao entendimento que os investigadores seniores entrevistados têm sobre os conceitos de Sustentabilidade e de IC, que:

i. Todos os investigadores entrevistados reconhecem a pertinência destes conceitos e sua transversalidade face aos desafios do mundo atual. No entanto, apenas alguns os identifiquem como conceitos transversais ao $\mathrm{CIE}$ em que estão integrados e poucos os assumem como conceitos-chave para a investigação que desenvolvem e/ou orientam;

ii. A maioria dos investigadores identifica Sustentabilidade e IC como valores inegáveis e grandes orientações e/ou metas no domínio da educação, entendida numa perspetiva mais humanista. Contudo, reconhecem que a sua explicitação nos projetos de investigação que desenvolveram e desenvolvem nem sempre foi valorizada. Para a maioria dos investigadores entrevistados, estes conceitos estão associados a domínios de investigação específicos - a Sustentabilidade ligada à investigação no domínio das ciências e a IC à investigação no domínio das línguas - pelo que não os sentem como conceitos-chave "seus".

iii. Grande parte destes investigadores refere-se a estes conceitos como complexos e multidimensionais. Alguns identificam mesmo algumas das dimensões que se podem considerar para a definição de Sustentabilidade e de IC. Porém, a grande maioria dos investigadores menciona os conceitos sem nunca os definir ou formular explicitamente. A referência ao conceito de sustentabilidade é feita, sobretudo, numa perspetiva ambiental (e, em alguns casos, social) evidenciando uma perceção simplista e fragmentada (próxima do senso comum). A IC é referida, especialmente, como o entendimento entre pessoas (de culturas diferentes, com línguas diferentes), o que demonstra, tal como no conceito de Sustentabilidade, um entendimento parcelar.

iv. Nenhum dos investigadores faz a distinção entre os conceitos de Sustentabilidade e DS, utilizando-os como sinónimos. No entanto, a utilização dos dois conceitos é sempre feita numa perspetiva de futuro, de meta (entendimento mais próximo do conceito de Sustentabilidade).

Reconhecendo o compromisso com a Agenda 2030 como global e absolutamente fundamental, os resultados da investigação permitem sugerir a necessidade de um maior envolvimento e responsabilização da comunidade de educadores e de investigadores em educação para com os ODS. Sendo os investigadores entrevistados investigadores seniores, com um percurso académico reconhecido e com responsabilidades na definição das políticas de investigação de um CIE, o entendimento que têm sobre 
os conceitos de sustentabilidade e de IC e sobre a relevância que estes têm para o campo da educação é absolutamente determinante para marcar a agenda de investigação em educação nos próximos anos. Apenas com o reconhecimento e valorização destes domínios por parte dos investigadores será possível integrar a Sustentabilidade e a IC na investigação em educação e contribuir efetivamente para as metas fixadas. É, então, fundamental:

v. Reconhecer a agenda 2030 enquanto meta global e assumir o compromisso de contribuir para a sua concretização, identificando, desde logo, relações com os domínios de investigação e possíveis contributos;

vi. Discutir e refletir, em grupos alargados e restritos, sobre os documentos orientadores desta agenda e sobre os seus conceitos chave (como os conceitos de sustentabilidade e de IC) e de como estes podem orientar o trabalho que se desenvolve ao nível da investigação e dos seus outputs, por um lado, e da formação de formadores, por outro (a criação de novas Unidades Curriculares, novas metodologias de trabalho e abordagem explícita e integrada de questões de sustentabilidade e IC são, apenas, alguns exemplos a considerar);

vii. Aproximar investigação e decisão política, divulgando de forma estratégica e clara, os resultados da investigação de modo a que estes possam servir de fundamentação a tomadas de decisão no âmbito das políticas educativas. Esta aproximação permitirá, também, que a investigação se ocupe de questões identificadas como problemáticas pelos decisores políticos, evidenciando a utilidade e aplicabilidade dos resultados da investigação;

viii. Criar grupos de trabalho internacionais e pluridisciplinares, onde tenham lugar parceiros provenientes de contextos não académicos, envolvendo, por exemplo, autarquias, empresas e ONG.

O pensamento aqui apresentado procurou descrever e analisar aquilo que pensam sobre os conceitos de Sustentabilidade e IC os membros mais qualificados e com mais responsabilidades de um CIE que tem um processo de investigação ativo e que se pensa em todo o momento. É por esta razão, também, um processo inacabado. Contudo, face à pertinência dos desafios lançados pela Agenda 2030 a todos os investigadores e a todas as estruturas de investigação, a avaliação das unidades de investigação e do pensamento dos investigadores que as integram surgem como exercícios fundamentais para o cumprimento dos ODS. Neste sentido, o artigo que se apresenta pretende ser um contributo para uma reflexão partilhada sobre a necessária reorientação da investigação em educação e para a emergência de um novo paradigma da investigação em educação orientado para a Sustentabilidade e IC. 
Nota: Este trabalho foi financiado pelo CIDTFF, projeto BPD/UI57/2390/2016 através de fundos nacionais da FCT-MCTES-PT.

\section{Referências Bibliográficas}

Alarcão, I. \& Araújo e Sá, M. H. (2010). Era uma vez... a Didática de Línguas em Portugal. Cadernos do LALE, Série Reflexões 3. Aveiro: Universidade de Aveiro, CIDTFF.

Amado, J. (2017). Introdução a Estratégias Gerais de Investigação: natureza e fundamentos. In J. Amado (Coord). Manual de Investigação Qualitativa em Educação (pp. 119-122). Coimbra: Imprensa da Universidade de Coimbra.

Amador, F. \& Oliveira, C. B. P. (2013). Integrating Sustainability into the University: Past, Present and Future. In S. Caeiro, W. Leal Filho. C. Jabbour, \& U. Azeiteiro (Eds). Sustainability Assessment Tools in Higher Education Institutions, 65-78. Switzerland: Springer.

Araújo e Sá, M. H. (2012). A intercompreensão em ação e em construção - dinâmicas na interação plurilingue. Lição de Síntese. Provas de Agregação. Não publicada. Universidade de Aveiro.

Bardin, L. (2014). Análise de Conteúdo. Lisboa: Edições 70.

Bettencourt, L. M. A. \& Kaur, J. (2011). Evolution and structure of sustainability science. Proceedings of the National Academy of Sciences, 108, (49). 19540-19545.

Bogdan, R. \& Biklen, S. (2013). Investigação qualitativa em Educação. Uma introdução à teoria e aos métodos. Porto: Porto Editora.

Constanza, R. \& Patten, B. C. (1995). Defining and predicting sustainability. Ecological Economics, 15, 193-196.

Costa, A. P. \& Amado, J. (2018). Análise de conteúdo suportada por software. Oliveira de Azeméis: Ludomedia.

Drexhage, J. and Murphy, D. (2010). Sustainable Development: From Brundtland to Rio 2012. In: United Nations Headquarters, 1st Meeting by the High Level Panel on Global Sustainability, United Nations, New York.

Faustino, M. \& Amador, F. (2016). O conceito de "Sustentabilidade": Migração e Mudanças de Significados no Âmbito Educativo. Indagatio Didactica, 8(1), 2021-2033.

Ferrão Tavares, C., Silva, J. \& Silva e Silva, M. (2010). Des notions actuelles (et potentielles) d'intercompréhension en didactique des langues-cultures. REDINTER-Intercompreensão. Revista da Rede Europeia sobre Intercompreensão, 1, 125-155.

Giovannonni, E. \& Fabietti, G. (2014). What is Sustainability? A review of the Concept and its applications. In C. Busco et al. (eds) (2014). Integrated Report, DOI. (21-40). Switzerland: Springer.

Melo, S. \& Santos, L. (2007). Intercompréhension(s): les multiples déclinaisons d'un concept. In F. Capucho, A. Martins, C. Degache \& M. Tost (Eds), Diálogos em Intercompreensão. Lisboa: Universidade Católica Editora.

Ministère de la Culture et de la Communication (2015). L'intercompréhension. Paris: Délégation générale à la langue française et aux langues de France. 
Pinho, A. S. \& Andrade, A. I. (2011). Intercultural narratives and Intercomprehension: steps to teachers' sensitivity towards diversity-oriented teaching. Comunicação em Across Languages and Cultures $4^{\text {th }}$ International Adriatic-Ionian Conference. Veneza, Ca' Foscari University of Venice.

Pipere, A. (2016). Envisioning Complexity: Towards a New Conceptualization of Educational Research for Sustainability. Discourse and Communication for Sustainable Education, 7(2), 68-91.

Sartori, S., Silva, F. \& Campos, L. (2014). Sustainability and Sustainable Development: a taxonomy in the field of literature. Ambiente e Sociedade, XVII (1), 1-20.

UN. Transforming our world. The 2030 Agenda for Sustainable Development. UN (A/RES/70/1).

UNESCO (2005). International Implementation Scheme. Paris: UNESCO

UNESCO (2012). Shaping the Education of Tomorrow - Abridge Version. Paris: UNESCO.

UNESCO (2014). The Global Action Programme on ESD. Paris: UNESCO.

UNESCO (2017). Education for Sustainable Development Goals. Learning Objectives. Paris: UNESCO.

Yin, R. K. (2001). Estudo de caso: planejamento e métodos. Porto Alegre: Bookman.

Patrícia Sá

Centro de Investigação Didática e Tecnologia na Formação de Formadores (CIDTFF)

Universidade de Aveiro

E-mail: f2390@ua.pt

J. Bernardino Lopes

Centro de Investigação Didática e Tecnologia na Formação de Formadores (CIDTFF) Universidade de Trás-os-Montes e Alto Douro (UTAD)

E-mail: blopes@utad.pt

Isabel P. Martins

Centro de Investigação Didática e Tecnologia na Formação de Formadores (CIDTFF)

Universidade de Aveiro

E-mail: imartins@ua.pt

\section{Correspondência}

Patrícia Sá

Centro de Investigação de Didática e Tecnologia na Formação de Formadores (CIDTFF)

Departamento de Educação da Universidade de Aveiro

Campus Universitário de Santiago

3810-193 Aveiro

Data de submissão: Setembro de 2017

Data de avaliação: Novembro de 2017

Data de publicação: Janeiro 2019 